\title{
TENSOR PRODUCTS OVER BANACH ALGEBRAS
}

\author{
BY \\ B. R. GELBAUM
}

0. Introduction. In [4], [5], [6] the structures $A_{1} \otimes_{\gamma} A_{2}$, where $A_{1}$ and $A_{2}$ are Banach algebras, are discussed. Actually, a proper parallel to the algebraic situation is: three commutative Banach algebras $A, B, C$, where $A$ and $B$ are $C$-bimodules (in the sense described below), and some Banach-algebraic version of $A \otimes_{C} B$. In the first part of the following, we shall give a general discussion of $A \otimes_{C} B$, a natural Banach-algebraic version of the (algebraic) tensor product of $A$ and $B$ over $C$. Thereafter, we shall discuss a special case in which $A, B, C$ are group algebras of locally compact abelian groups. Finally we shall handle the even more special problem in which $A$ and $B$ are group algebras of locally compact abelian groups and $C$ is a group algebra of a compact abelian group. In the last two parts, particularly the last, a connection will be established between the theory of tensor products and the theory of group extensions. In this connection, the author thanks L. Auslander for indicating the point in question. The author is also indebted to Dr. B. Natzitz for pointing out errors and the need for clarifying several items of an earlier draft of this paper.

1. $A \otimes_{C} B$. In analogy to the treatment in [5], [6] we consider three commutative Banach algebras $A, B, C$ where it is assumed that $A$ and $B$ are $C$-bimodules. The fundamental topological assumption is: For $(a, b, c) \in A i \times B \times C$, $\|a c\| \leqq\|a\|\|c\|$ and $\|b c\| \leqq\|b\|\|c\|$.

We construct the linear function space and commutative topological algebra

$$
F_{C}(A, B)=\left\{f \mid f \in C^{A \times B}, \gamma_{1}(f) \equiv \sum_{(a, b)}\|f(a, b)\|\|a\|\|b\|<\infty\right\}
$$

where multiplication $*$ of two elements $f_{1}, f_{2}$ is defined by

$$
\begin{aligned}
\left(f_{1} * f_{2}\right)(a, b) & =\sum_{a_{1} a_{2}=a ; b_{1} b_{2}=b} f_{1}\left(a_{1}, b_{1}\right) f_{2}\left(a_{2}, b_{2}\right), & & \text { if }\|a\|\|b\|>0, \\
& =0, & & \text { otherwise. }
\end{aligned}
$$

The relations

$$
\begin{aligned}
& \sum_{(a, b)}\left(\sum_{a_{1} a_{2}=a ; b_{1} b_{2}=b}\left\|f_{1}\left(a_{1}, b_{1}\right) f_{2}\left(a_{2}, b_{2}\right)\right\|\right)\|a\|\|b\| \\
& \quad \leqq \sum_{a_{1}, a_{2}, b_{1}, b_{2}}\left\|f_{1}\left(a_{1}, b_{1}\right)\right\|\left\|f_{2}\left(a_{2}, b_{2}\right)\right\|\left\|a_{1}\right\|\left\|a_{2}\right\|\left\|b_{1}\right\|\left\|b_{2}\right\| \\
& \quad=\gamma_{1}\left(f_{1}\right) \gamma_{1}\left(f_{2}\right)<\infty
\end{aligned}
$$

Received by the editors July 5, 1962 and, in revised form, February 7, 1964. 
show that the series defining $\left(f_{1} * f_{2}\right)(a, b)$ converges whenever $\|a\|\|b\|>0$. Thus $f_{1} * f_{2}$ is well defined and is in $F_{C}(A, B)$. Commutativity and associativity of $*$ are easily proved.

In $F_{C}$ we consider the closed ideal $I$ generated by the functions of the following types:

$$
\begin{aligned}
f\left(a_{1}+a_{2}, b_{1}\right) & =-f\left(a_{1}, b_{1}\right)=-f\left(a_{2}, b_{1}\right), \\
f(a, b) & =0, \text { otherwise } \\
f\left(a_{1}, b_{1}+b_{2}\right) & =-f\left(a_{1}, b_{1}\right)=-f\left(a_{1}, b_{2}\right), \\
f(a, b) & =0, \text { otherwise; } \\
f\left(a_{1} c_{1}, b_{1}\right) & =-f\left(a_{1}, c_{1} b_{1}\right), \\
f(a, b) & =0, \text { otherwise; } \\
f\left(a_{1} c_{1}, b_{1}\right) c_{1} & =-f\left(a_{1}, b_{1}\right), \\
f(a, b) & =0, \text { otherwise. }
\end{aligned}
$$

(If $C$ has no identity, then for $\lambda \in C$ we include in $I$ the functions

$$
\begin{aligned}
f\left(a_{1} \lambda, b_{1}\right) & =-f\left(a_{1}, \lambda b_{1}\right), \\
f(a, b) & =0, \text { otherwise } \\
f\left(a_{1} \lambda, b_{1}\right) \lambda & =-f\left(a_{1}, b_{1}\right), \\
f(a, b) & =0, \text { otherwise. })
\end{aligned}
$$

We then define $A \otimes_{C} B$ to be $F_{C}(A, B) / I$. After the next remarks we shall show that $D=A \otimes_{C} B$ is a commutative Banach algebra and then we shall investigate the maximal ideal space $\mathfrak{M}_{D}$.

It is of interest first to illustrate the above concepts.

Example 1. Let $S \equiv\{z|| z \mid \leqq 1\}$ and let $A=B=C(S)$. Let $C=A_{1}$ $\equiv\left\{f \mid f\right.$ analytic in $S^{0}, f$ continuous on $\left.S\right\}$. If $A$ and $B$ are regarded as $C$-bimodules with elements of $C$ acting by pointwise multiplication, then the conditions of our general discussion prevail and $A \otimes_{C} B$ is definable.

Example 2. Let $G, H, K$ be locally compact abelian groups, and let $\theta_{G}: K \rightarrow G, \theta_{H}: K \rightarrow H$ be homomorphisms such that $\theta_{G}(K)$ and $\theta_{H}(K)$ are closed subgroups of $G$ and $H$ respectively. Let $A=L_{1}(G), B=L_{1}(H)$ and $C=L_{1}(K)$ and for $a \in A, c \in C$ define $a c$ by

$$
a c(\xi)=\int_{K} a\left(\xi-\theta_{G}(\zeta)\right) c(\zeta) d \zeta .
$$

Again (see $\S 3$ ) the definitions permit the construction of $A \otimes_{C} B$. (Note the case: $G, H$ subgroups of a supergroup, $G \supset K, H \supset K, \theta_{G}, \theta_{H}$ the inclusion maps.) 
Example 3. Let $H^{\infty}=\left\{f \mid f\right.$ analytic in $S^{0}$, $\left.\sup _{S^{0}}|f|<\infty\right\}$. Then if (a) $A=B=C(S), C=H^{\infty}$ or if (b) $A=B=H^{\infty}$ and $C=A_{1}, A \otimes_{C} B$ is definable in a standard way.

Example 4. Let $C^{(k)}([0,1])$ be the set of functions continuous on $[0,1]$ and having $k$ continuous derivatives on $[0,1]$. For $f \in C^{(k)}([0,1])$ let

$$
\|f\|=\sum_{j=1}^{k} \sup \left\{\left|f^{(j)}(x)\right| \mid 0 \leqq x \leqq 1\right\} .
$$

Then with the obvious definitions of operations, if $0 \leqq k_{1}, k_{2} \leqq k_{3}$ and if $A=C^{\left(k_{1}\right)}([0,1]), \quad B=C^{\left(k_{2}\right)}([0,1]), C=C^{\left(k_{3}\right)}([0,1])$, we see that $A \otimes_{C} B$ is definable.

Example 5. $A$ and $B$ are two commutative subalgebras of a commutative Banach algebra and $C=A \cap B$.

In defining $A \otimes_{c} B=D$, we did not prove it is a Banach space. However, the only question of substance is resolved by

LeMma 1. If $f \in F_{C}(A, B)$ and if $\gamma_{1}(f)=0$, then $f \in I$.

Proof. For each pair $(a, b)$ either $\|a\|=0$ or $\|b\|=0$ or $f(a, b)=0$. We may confine ourselves to the case $\|a\|=0$. Let $f(a, b)=c$ and choose $a_{1} \neq 0$. Then using the customary symbolism we find:

$$
\begin{aligned}
c\left(\left(a_{1}-a_{1}\right) \otimes b\right) & -c\left(a_{1} \otimes b\right)-c\left(\left(-a_{1}\right) \otimes b\right) \in I, \quad \operatorname{via}(1) ; \\
& -c\left(a_{1} \otimes b\right)-c\left(\left(-a_{1}\right) \otimes b\right) \in I, \quad \operatorname{via}\left(4^{\prime}\right) ;
\end{aligned}
$$

and thus $c(0 \otimes b) \in I$.

Thus, from Lemma 1 we can conclude that if $\gamma(z)$ denotes the quotient seminorm of $z \in D$, then $\gamma(z)=0$ if and only if $z=0$. It is now clear that $D$ is a commutative Banach algebra.

2. Maximal ideals. Let $\mathfrak{M}_{C}$ be the set of multiplicative homomorphisms of $C$ into $C$ (or, equivalently, the set of regular maximal ideals of $C$ ). Let $\mathfrak{M}_{C}^{0}$ be $\mathfrak{M}_{C}$ with the null map adjoined. We use $\mathfrak{M}_{A}$ and $\mathfrak{M}_{B}$ in a similar sense.

THEOREM 1. There is a homeomorphism $\tau: \mathfrak{M}_{D} \rightarrow \mathfrak{M}_{A} \times \mathfrak{M}_{B}$ and the image of $\tau$ is a locally compact subset of $\mathfrak{M}_{A} \times \mathfrak{M}_{B}$. There are continuous mappings $\mu: \mathfrak{M}_{A} \rightarrow \mathfrak{M}_{C}^{0}, v: \mathfrak{M}_{B} \rightarrow \mathfrak{M}_{C}^{0}$ such that if $\rho=\mu \times v$ then $\tau\left(\mathfrak{M}_{D}\right)=\rho^{-1}(\Delta)$ where $\Delta=$ diagonal of $\mathfrak{M}_{C} \times \mathfrak{M}_{C}$. These mappings are furthermore related as follows: If $z \in D$ and if $z$ has the representative

$$
\sum_{n=1}^{\infty} c_{n}\left(a_{n} \otimes b_{n}\right) \quad[5],[6],
$$

then for any $M_{D} \in \mathfrak{M}_{D}$ 


$$
\hat{z}\left(M_{D}\right)=\sum_{n=\mathbf{i}}^{\infty} \hat{c}_{n}\left(M_{C}\right) \hat{a}_{n}\left(M_{A}\right) \hat{b}_{n}\left(M_{B}\right)
$$

where $\tau\left(M_{D}\right)=\left(M_{A}, M_{B}\right)$ and $\mu\left(M_{A}\right)=v\left(M_{B}\right)=M_{C}$.

The proof of the theorem will follow a sequence of lemmas.

LEMMA 1. There is a function $k\left(c, M_{A}\right)$, independent of a such that for $a \in A,[(c a)]^{\wedge}\left(M_{A}\right)=k\left(c, M_{A}\right) \hat{a}\left(M_{A}\right)$.

Proof. Let $a \notin M_{A}$ and let

$$
k\left(c, a, M_{A}\right)=[(c a)]^{\wedge}\left(M_{A}\right) / \hat{a}\left(M_{A}\right) .
$$

We show that so long as $a \notin M_{A}, k\left(c, a, M_{A}\right)$ is independent of $a$. Indeed, if $a_{1} \notin M_{A}$,

$$
k\left(c, a, M_{A}\right)-k\left(c, a_{1}, M_{A}\right)=\frac{[(c a)]^{\wedge}\left(M_{A}\right) \hat{a}_{1}\left(M_{A}\right)-\left[\left(c a_{1}\right)\right]^{\wedge}\left(M_{A}\right) \hat{a}\left(M_{A}\right)}{\hat{a}\left(M_{A}\right) \hat{a}_{1}\left(M_{A}\right)} .
$$

The numerator of the last fraction is $\left[\left(c a a_{1}\right)\right]^{\wedge}\left(M_{A}\right)-\left[\left(c a_{1} a\right)\right]^{\wedge}\left(M_{A}\right)$ which is 0 since $A$ is commutative. Dropping $a$ from $k\left(c, a, M_{A}\right)$, we let the result denote the required function: $k\left(c, M_{A}\right)$. If $\hat{a}\left(M_{A}\right) \neq 0$, then clearly $[c a]^{\wedge}\left(M_{A}\right)=k\left(c, M_{A}\right) \hat{a}\left(M_{A}\right)$.

We observe that every $M_{A} \in \mathfrak{M}_{A}$ is a $C$-ideal and similarly every $M_{B} \in \mathfrak{M}_{B}$ is a $C$-ideal. Indeed, if $a \in M_{A}, c \in C$, let $a_{n} \notin M_{A}, a_{n} \rightarrow a$. Then $c a_{n} \rightarrow c a,\left[c a_{n}\right]^{\wedge}\left(M_{A}\right)=k\left(c, M_{A}\right) \hat{a}_{n}\left(M_{A}\right) \rightarrow 0$, i.e., $[c a]^{\wedge}\left(M_{A}\right)=0$.

Hence, in particular, for all $a:[c a]^{\wedge}\left(M_{A}\right)=k\left(c, M_{A}\right) \hat{a}\left(M_{A}\right)$.

Corollary. If for some $a_{0} \notin M_{A}, c a_{0} \in M_{A}$, then $c A \subset M_{A}$.

Proof. $k\left(c, M_{A}\right)=0$, whence for any $a \in A,[c a]^{\wedge}\left(M_{A}\right)=0$.

LeMma 2. For $M_{A}$ fixed, $k\left(c, M_{A}\right)$ is a continuous homomorphism of $C$ into $C$. (Either there is an $M_{C} \in \mathfrak{M}_{C}$ such that $\hat{c}\left(M_{C}\right)=k\left(c, M_{A}\right)$ or $k\left(c, M_{A}\right) \equiv 0$.)

Proof. The equations $k\left(c_{1}+c_{2}, M_{A}\right)=k\left(c_{1}, M_{A}\right)+k\left(c_{2}, M_{A}\right)$ and $k\left(\lambda c_{1}, M_{A}\right)$ $=\lambda k\left(c_{1}, M_{A}\right), \lambda \in C$, are clearly true. On the other hand $\left[\left(c_{1} c_{2} a\right)\right]^{\wedge}\left(M_{A}\right)=k\left(c_{1}, M_{A}\right)$ $\left(c_{2} a\right)^{\wedge}\left(M_{A}\right)=k\left(c_{1}, M_{A}\right) k\left(c_{2}, M_{A}\right) \hat{a}\left(M_{A}\right)$, whence $k\left(c_{1} c_{2}, M_{A}\right)=k\left(c_{1}, M_{A}\right) k\left(c_{2}, M_{A}\right)$. Finally, if $\lim _{n \rightarrow \infty} c_{n}=0$, then for $a \notin M_{A}$

$$
\left|k\left(c_{n}, M_{A}\right)\right| \leqq \frac{\left\|c_{n}\right\|\|a\|}{\hat{a}\left(M_{A}\right)} \rightarrow 0
$$

as $n \rightarrow \infty$. Thus $k\left(c, M_{A}\right)$ is continuous in $c$.

Note that if $C=C$ then $k\left(c, M_{A}\right)=c$.

Defining $\mu: \mathfrak{M}_{A} \rightarrow \mathfrak{M}_{C}^{0}$ by $\mu\left(M_{A}\right)=M_{C}$ if $k\left(c, M_{A}\right)=\hat{c}\left(M_{C}\right)$ and $\mu\left(M_{A}\right)=$ null map if $k\left(c, \mathfrak{M}_{A}\right) \equiv 0$, and similarly defining $v: \mathfrak{M}_{B} \rightarrow \mathfrak{M}_{C}^{0}$, we shall define $\tau$ so that $\tau, \mu, v$ behave as stated in the theorem. (In Example 5, if $C$ is contained in 
the radicals of both algebras $A$ and $B$, then $\mu\left(M_{A}\right) \equiv$ null map, $v\left(M_{B}\right) \equiv$ null map for all $M_{A}, M_{B}$. In other words $\rho\left(\mathfrak{M}_{A} \times \mathfrak{M}_{B}\right)$ may be completely outside $\mathfrak{M}_{C} \times \mathfrak{M}_{C}$.) First we prove

LEMMA 3. $\mu$ and $v$ are continuous.

Proof. Let $\mu\left(M_{A}\right)=X$, where $X$ is either some $M_{C}$ or $X$ is the null map. If $N(X)=\left\{Y \in \mathfrak{M}_{c}^{0}|| \hat{c}_{i}(Y)-\hat{c}_{i}(X) \mid<\varepsilon, \quad i=1,2, \cdots, n\right\}$, let $\hat{a}\left(M_{A}\right)=1$ and let $N\left(M_{A}\right)=\left\{M_{A}^{\prime}||\left[\left(a c_{i}\right)\right]^{\wedge}\left(M_{A}^{\prime}\right)-\left[\left(a c_{i}\right)\right]^{\wedge}\left(M_{A}\right)\left|<\varepsilon_{1},\right| \hat{a}\left(M_{A}^{\prime}\right)-\hat{a}\left(M_{A}\right) \mid<\varepsilon_{1}\right.$, $i=1,2, \cdots, n\}$. Then for $0<\varepsilon_{1}<\varepsilon /\left(1+\max _{i}\left\|c_{i}\right\|\right), \mu\left(N\left(M_{A}\right)\right) \subset N(X)$. Hence $\mu$ (and similarly $v$ ) is continuous.

To define $\tau$ we proceed as follows. Let $M_{D} \in \mathfrak{M}_{D}$ and let $u \in D$ be such that $\hat{u}\left(M_{D}\right)=1$. Regarding $D$ as a bimodule with respect to $A, B$ and $C$ we define:

$$
\begin{aligned}
& \phi_{A}(a)=[(a u)]^{\wedge}\left(M_{D}\right), \\
& \phi_{B}(b)=[(b u)]^{\wedge}\left(M_{D}\right) \text {, } \\
& \phi_{C}(c)=[(c u)]^{\wedge}\left(M_{D}\right) \quad[5],[6] \text {. }
\end{aligned}
$$

These are clearly continuous homomorphisms into $C$. We shall show that $\phi_{A}$ and $\phi_{B}$ are surjections by first noting

LEMma 4. A regular ideal in $D$ is simultaneously an $A$-ideal, B-ideal and C-ideal.

Proof. See proof of Lemma $1[5, \S 1]$.

If $\phi_{A}^{-1}(0)=A$, and if $u$ is represented by

$$
\sum_{n=1}^{\infty} c_{n}^{\prime}\left(a_{n}^{\prime} \otimes b_{n}^{\prime}\right),
$$

then, for any $a_{m}^{\prime}$,

$$
\sum_{n=1}^{\infty} c_{n}^{\prime}\left(a_{n}^{\prime} a_{m}^{\prime} \otimes b_{n}^{\prime}\right)
$$

represents an element in $M_{D}$, whence $u^{2} \in M_{D}$ and a contradiction results. Thus let $\phi_{A}^{-1}(0)=M_{A}, \phi_{B}^{-1}(0)=M_{B}, \phi_{C}^{-1}(0)=X$ (where $X \in \mathfrak{M}_{C}^{0}$ ). We shall show $\mu\left(M_{A}\right)=v\left(M_{B}\right)=X$. Indeed, since $u^{2}-u \in M_{D}$, we see that for $a \in A, b \in B, c \in C$.

$$
\begin{aligned}
{[(a b c u)]^{\wedge}\left(M_{A}\right) } & =[(a c u)]^{\wedge}\left(M_{A}\right)[(b u)]^{\wedge}\left(M_{A}\right) \\
& =\phi_{A}(a c) \phi_{B}(b)
\end{aligned}
$$

and

$$
\begin{aligned}
\phi_{A}(a c)=[(a c u)]^{\wedge}\left(M_{D}\right) & =[(a u)]^{\wedge}\left(M_{D}\right)[(c u)]^{\wedge}\left(M_{D}\right) \\
& =\phi_{A}(a) \phi_{C}(c) .
\end{aligned}
$$


Thus, if $\phi_{A}(a) \neq 0, \phi_{A}(a c) / \phi_{A}(a)=\phi_{C}(c)$. Similarly $\phi_{B}(b c)=\phi_{B}(b) \phi_{C}(c)$. In summary, for $z \in D$, and $z$ represented by

$$
\sum_{n=1}^{\infty} c_{n}\left(a_{n} \otimes b_{n}\right)
$$

we have the formula

$$
\hat{z}\left(M_{D}\right)=\sum_{n=1}^{\infty} \hat{c}_{n}(X) \hat{a}_{n}\left(M_{A}\right) \hat{b}_{n}\left(M_{B}\right) .
$$

Note that in the above $X \in \mathfrak{M}_{C}$, since otherwise $\hat{z}\left(M_{D}\right)=0$ for all $z$, and this contradicts the fact that $M_{D} \in \mathfrak{M}_{D}$. Thus we see that an $M_{D} \in \mathfrak{M}_{D}$ engenders a pair $\left(M_{A}, M_{B}\right) \in \mathfrak{M}_{A} \times \mathfrak{M}_{B}$ such that $\rho\left(M_{A}, M_{B}\right) \in \Delta$. (Referring again to Example 5 , we see that if $C$ is contained in the radicals of both algebras $A$ and $B$, then $\mathfrak{M}_{D}$ is empty.) Clearly if $\left(M_{A}, M_{B}\right) \in \rho^{-1}(\Delta)$ and if $\mu\left(M_{A}\right)=v\left(M_{B}\right)=M_{C}$ then the mapping taking $z \in D$ into

$$
\sum_{n=1}^{\infty} \hat{c}_{n}\left(M_{C}\right) \hat{a}_{n}\left(M_{A}\right) \hat{b}_{n}\left(M_{B}\right)
$$

(where $z$ is represented by $\sum_{n=1}^{\infty} c_{n}\left(a_{n} \otimes b_{n}\right)$ ) provides a nontrivial homomorphism $D \rightarrow C$. Let $\tau\left(M_{D}\right)=\left(M_{A}, M_{B}\right)$. We must now show that $\tau$ is a homeomorphism.

$\tau$ is 1-1: If $\tau\left(M_{D}\right)=\tau\left(M_{D}^{\prime}\right)$, then for any $z \in D$ represented by $c(a \otimes b)$ we have $\hat{z}\left(M_{D}\right)=\hat{z}\left(M_{D}^{\prime}\right)$, whence by linear extension, for any $z \in D, \hat{z}\left(M_{D}\right)=\hat{z}\left(M_{D}^{\prime}\right)$, and thus $M_{D}=M_{D}^{\prime}$. Bicontinuity of $\tau$ is established as in [4] and in the corrected version in [5].

REMARK. $\overline{\rho^{-1}(\Delta)} \subset \rho^{-1}(\Delta) \cup\left\{\left(M_{A}, M_{B}\right) \mid \mu\left(M_{A}\right)=v\left(M_{B}\right)=\right.$ null map $\}$. Indeed, if $\left(M_{A}, M_{B}\right) \in \overline{\rho^{-1}(\Delta)}$ and $\mu\left(M_{A}\right) \neq$ null map or $v\left(M_{B}\right) \neq$ null map, we may assume $\mu\left(M_{A}\right) \neq v\left(M_{B}\right)$ and $\mu\left(M_{A}\right) \neq$ null map. Thus we may choose $c \in v\left(M_{B}\right) \backslash \mu\left(M_{A}\right)$ and so that $\hat{c}\left(\mu\left(M_{A}\right)\right)=1$. Let $\hat{a}\left(M_{A}\right)=\hat{b}\left(M_{B}\right)=1$ and choose

$$
\begin{aligned}
N\left(M_{A}, M_{B}\right)=\left\{\left(M_{A}^{\prime}, M_{B}^{\prime}\right)|| \hat{a}\left(M_{A}\right)-\hat{a}\left(M_{A}^{\prime}\right)|<\varepsilon,| \hat{b}\left(M_{B}\right)-\hat{b}\left(M_{B}^{\prime}\right) \mid<\varepsilon,\right. \\
\left.\left|[(c a)]^{\wedge}\left(M_{A}^{\prime}\right)-[(c a)]^{\wedge}\left(M_{A}^{\prime}\right)\right|<\varepsilon,\left|[(c b)]^{\wedge}\left(M_{B}\right)-[(c b)]^{\wedge}\left(M_{B}^{\prime}\right)\right|<\varepsilon\right\} .
\end{aligned}
$$

Since $N\left(M_{A}, M_{B}\right) \cap \rho^{-1}(\Delta) \neq \varnothing$, there is in $N\left(M_{A}, M_{B}\right)$ a pair $\left(M_{A}^{\prime}, M_{B}^{\prime}\right)$ such that $\mu\left(M_{A}^{\prime}\right)=v\left(M_{B}^{\prime}\right)=M_{C}^{\prime} \neq$ null map. However $[(c a)]^{\wedge}\left(M_{A}\right)=1,([c b)]^{\wedge}\left(M_{B}\right)=0$,

$$
\left|\hat{a}\left(M_{A}^{\prime}\right)\right|<1+\varepsilon, \quad\left|\hat{b}\left(M_{B}^{\prime}\right)\right|>1-\varepsilon .
$$

Hence, by direct calculation $(1-\varepsilon) /(1+\varepsilon)<\left|\hat{c}\left(M_{C}^{\prime}\right)\right|<\varepsilon /(1-\varepsilon)$, an impossibility if $0<\varepsilon<1 / 3$. Thus $\overline{\rho^{-1}(\Delta)} \subset \rho^{-1}(\Delta) \cup\left\{\left(M_{A}, M_{B}\right) \mid \mu\left(M_{A}\right)=v\left(M_{B}\right)=\right.$ null map $\}$.

We have noted earlier, in connection with Example 5 , that $\rho\left(\mathfrak{M}_{A} \times \mathfrak{M}_{B}\right)$ may 
be $\left(\mathfrak{M}_{C}^{0} \backslash \mathfrak{M}_{\mathcal{C}}\right) \times\left(\mathfrak{M}_{C}^{0} \backslash \mathfrak{M}_{C}\right)$, i.e., the null maps. Hence $\rho\left(\mathfrak{M}_{A} \times \mathfrak{M}_{B}\right)$ does not necessarily contain $\Delta$. However we can prove

Propostion 1. $\rho\left(\mathfrak{M}_{A} \times \mathfrak{M}_{B}\right) \supset \Delta$ if and only if for every $M_{C} \in \mathfrak{M}_{C}$, $I\left(M_{C}\right)=\left\{z \mid z \in D, z\right.$ represented by $\left.\sum_{n=1}^{\infty} c_{n}\left(a_{n} \otimes b_{n}\right), c_{n} \in M_{C}, n=1,2, \cdots\right\}$ is contained in some $M_{D} \in \mathfrak{M}_{D}$, i.e., there is a pair $\left(M_{A}, M_{B}\right)$ such that $\mu\left(M_{A}\right)=v\left(M_{B}\right)$ $=M_{C}$ if and only if $I\left(M_{C}\right)$ is contained in some $M_{D} \in \mathfrak{M}_{D}$.

Proof. Assume $I\left(M_{C}\right)$ is contained in some $M_{D} \in \mathfrak{M}_{D}: I\left(M_{C}\right) \subset M_{D}$. Let $\tau\left(M_{D}\right)=\left(M_{A}, M_{B}\right), \mu\left(M_{A}\right)=v\left(M_{B}\right)=M_{C}^{\prime}$. We show $M_{C}=M_{C}^{\prime}$. If $M_{C} \neq M_{C}^{\prime}$, choose $c \in M_{C} \backslash M_{C}^{\prime}, a \notin M_{A}, b \notin M_{B}$. Then $w$ represented by $c(a \otimes b)$ satisfies

$$
\hat{w}\left(M_{D}\right)=\hat{c}\left(M_{C}^{\prime}\right) \hat{a}\left(M_{A}\right) \hat{b}\left(M_{B}\right) \neq 0 .
$$

On the other hand $w \in M_{D}$ and a contradiction results.

If $\mu\left(M_{A}\right)=v\left(M_{B}\right)=M_{C}$, let $M_{D}=\tau^{-1}\left(M_{A}, M_{B}\right)$. Then if $z$, represented by

$$
\sum_{n=1}^{\infty} c_{n}\left(a_{n} \otimes b_{n}\right)
$$

is in $I\left(M_{C}\right)$,

$$
\hat{z}\left(M_{D}\right)=\sum_{n=1}^{\infty} \hat{c}_{n}\left(M_{C}\right) \hat{a}_{n}\left(M_{A}\right) \hat{b}_{n}\left(M_{B}\right)=0
$$

Hence $I\left(M_{C}\right) \subset M_{D}$.

If $A, B, C$ are as in Example 5, then for $M_{D} \in \mathfrak{M}_{D}, \tau\left(M_{D}\right)=\left(M_{A}, M_{B}\right)$ where $\mu\left(M_{A}\right)=v\left(M_{B}\right)=M_{C}$ and $M_{C}$ is the kernel of both homomorphisms $A \rightarrow A / M_{A}$, $B \rightarrow B / M_{B}$ when they are confined to $C$. In fact $[(c a)]^{\wedge}\left(M_{A}\right)=\hat{c}\left(M_{A}\right) \hat{a}\left(M_{A}\right)$. Thus $k\left(c, M_{A}\right)=\hat{c}\left(M_{A}\right)$ and we see $\hat{c}\left(M_{C}\right)=\hat{c}\left(M_{A}\right)$. Similarly, $\hat{c}\left(M_{C}\right)=\hat{c}\left(M_{B}\right)$.

The discussion of the semisimplicity of $D$ when $A, B, C$ are semisimple will not be given. The case where $C=C$ is to the writer's knowledge unresolved and $a$ fortiori the general case remains an open problem. We shall see later how the semisimplicity of $D$ enters in an essential fashion in the study of the area associated with Example 2.

3. Group algebras. We now turn to a more detailed discussion of Example 2. Direct integration computations show that $A$ and $B$ are $C$-bimodules in the sense discussed. We shall in this section write $\hat{G}, \hat{H}, \hat{K}$ for $\mathfrak{M}_{A}, \mathfrak{M}_{B}, \mathfrak{M}_{C}$ and $\alpha, \beta, \gamma$ for $M_{A}, M_{B}, M_{C}$ [10]. One purpose of the development will be the discussion of the relationship of $D$ to the group algebra $L_{1}(\mathfrak{G})$ of a group $(5)$ constructible from $G, H, K$. To this end we proceed as follows:

Lemma 1. The maps $\mu: \hat{G} \rightarrow \hat{K}$ and $v: \hat{H} \rightarrow \hat{K}$ are the duals $\theta_{G}^{*}$ amd $\theta_{H}^{*}$ of the maps $\theta_{G}$ and $\theta_{H}$. 
Proof.

$$
\begin{aligned}
{[c a]^{\wedge}(\alpha) } & =\hat{c}(\mu(\alpha)) \hat{a}(\alpha) \\
& =\int_{G} \int_{K} a\left(\xi-\theta_{G}(\zeta)\right) c(\zeta) \overline{(\xi, \alpha)} d \zeta d \xi \\
& =\hat{a}(\alpha) \hat{c}\left(\theta_{G}^{*}(\alpha)\right) .
\end{aligned}
$$

Thus $\theta_{G}^{*}(\alpha)=\mu(\alpha)$ and similarly $\theta_{H}^{*}(\beta)=v(\beta)$.

It follows that

$$
\begin{aligned}
\tau\left(\mathfrak{M}_{D}\right) & =\left\{(\alpha, \beta) \mid \theta_{G}^{*}(\alpha)=\theta_{H}^{*}(\beta)\right\} \\
& =\left(\theta_{G}^{*} \times \theta_{H}^{*}\right)^{-1}(\text { diagonal }(\hat{K} \times \hat{K})),
\end{aligned}
$$

a closed subgroup of $\hat{G} \times \hat{H}$. If indeed $D=L_{1}(\mathfrak{H})$ for some locally compact abelian group $\mathfrak{G}$ then $\mathfrak{M}_{D}$ and $\hat{\mathfrak{G}}$ are identifiable and $\mathfrak{M}_{D}=\hat{\mathfrak{G}}$. Since $\mathfrak{M}_{D}$ is already exhibited as a group we find

$$
\hat{\mathfrak{M}}_{D} \cong(G \times H) / \mathfrak{M}_{D}^{\perp}
$$

where $\mathfrak{M}_{D}^{\perp}=\left\{(\xi, \eta) \mid((\alpha, \beta),(\xi, \eta))=1\right.$, all $\left.(\alpha, \beta) \in \mathfrak{M}_{D}\right\}$ and the candidate for $(5$ is $(G \times H) / \mathfrak{M}_{D}^{\perp}$.

LEMMA 2. Let $\tilde{\theta}_{H}: K \rightarrow H$ denote the mapping $\zeta \rightarrow-\theta_{H}(\zeta)$. Then $\mathfrak{M}_{D}^{\perp}=\left(\theta_{G} \times \tilde{\theta}_{H}\right)($ diagonal $(K \times K)) \equiv Q$.

Proof. $Q$ is a closed subgroup of $G \times H$. For if for some net $\left(\theta_{G}\left(\zeta_{\rho}\right), \tilde{\theta}_{H}\left(\zeta_{\rho}\right)\right) \rightarrow(\xi, \eta) \in G \times H$, then from the fact that $\theta_{G}(K)$ and $\tilde{\theta}_{H}(K)$ are closed and the mappings $\theta_{G}: K \rightarrow \theta_{G}(K), \tilde{\theta}_{I I}: K \rightarrow \tilde{\theta}_{H}(K)$ are homomorphisms, hence open, we conclude $(\xi, \eta) \in \theta_{G}(K) \times \tilde{\theta}_{H}(K)$. Let $\theta_{G}\left(\zeta_{1}\right)=\xi, \tilde{\theta}_{H}\left(\zeta_{2}\right)=\eta$. Using the fact that $\theta_{G}: K \rightarrow \theta_{G}(K), \tilde{\theta}_{H}: K \rightarrow \tilde{\theta}_{H}(K)$ are open, we can find a subnet $\zeta_{\sigma_{1}}^{\prime} \rightarrow \zeta_{1}$, and a subsubnet $\zeta_{\sigma_{2}}^{\prime \prime} \rightarrow \zeta_{2}$ such that $\theta_{G}\left(\zeta_{\sigma_{1}}^{\prime}\right) \rightarrow \xi, \tilde{\theta}_{H}\left(\zeta_{\sigma_{2}}^{\prime \prime}\right) \rightarrow \eta$. Since $\zeta_{\sigma_{1}}^{\prime \prime}$ is a subsubnet of $\zeta_{\sigma_{2}}^{\prime}$, and $K$ is a $T_{2}$-space, we conclude $\zeta_{1}=\zeta_{2}$ and $(\xi, \eta) \in Q$.

Now if $\xi=\theta_{G}(\zeta), \eta=\tilde{\theta}_{H}(\zeta)$, then for $(\alpha, \beta) \in \mathfrak{M}_{D}$

$$
\left(\theta_{G}(\zeta), \alpha\right)\left(\tilde{\theta}_{H}(\zeta), \beta\right)=\left(\zeta, \theta_{G}^{*}(\alpha)\right) \overline{\left(\zeta, \theta_{H}^{*}(\beta)\right)}=1 .
$$

Hence $Q \subset \mathfrak{M}_{D}^{\perp}$. On the other hand, if $(\xi, \eta) \in \mathfrak{M}_{D}^{\perp} \backslash Q$, choose $\left(\alpha_{0}, \beta_{0}\right)$ such that $\left(\xi, \alpha_{0}\right)\left(\eta, \beta_{0}\right) \neq 1$ and $\left(\theta_{G}(\zeta), \alpha_{0}\right)\left(\tilde{\theta}_{H}(\zeta), \beta_{0}\right)=1$, all $\zeta \in K$. Then for all $\alpha, \beta$ such that $\theta_{G}^{*}(\alpha)=\theta_{H}^{*}(\beta)$ (i.e., $\left.(\alpha, \beta) \in \mathfrak{M}_{D}\right)$

$$
(\xi, \alpha)(\eta, \beta)=1 \text {. }
$$

But, by assumption $\left(\zeta, \theta_{G}^{*}\left(\alpha_{0}\right)\right)=\left(\zeta, \theta_{H}^{*}\left(\beta_{0}\right)\right)$, all $\zeta \in K$. Hence $\theta_{G}^{*}\left(\alpha_{0}\right)=\theta_{H}^{*}\left(\beta_{0}\right)$ and thus $\left(\xi, \alpha_{0}\right)\left(\eta, \beta_{0}\right)=1$, a contradiction. Thus $\mathfrak{M}_{D}^{\perp}=Q$. The mapping $\theta_{G} \times \tilde{\theta}_{H}$ : diagonal $(K \times K) \rightarrow \mathfrak{M}_{D}^{\perp}$ is easily shown to be continuous and open. (Here and earlier we have exploited the standard proposition Let $\Phi: X \rightarrow Y$ be: 
a surjection. Then $\Phi$ is open if and only if whenever $y_{\rho}=\Phi\left(x_{\rho}\right) \rightarrow \Phi(x)=y$ for some nets $y_{\rho}, x_{\rho}$, there is a net $x_{\sigma}^{\prime} \rightarrow x$ and a subnet $y_{\rho(\sigma)}^{\prime}$ of $y_{\rho}$ such that $\Phi\left(x_{\sigma}^{\prime}\right)$ $=y_{\rho(\gamma)} \rightarrow y$.) Manifestly $\theta_{G} \times \tilde{\theta}_{H}$ is a homomorphism.

Presumably then $\mathfrak{F}_{\text {s }}$ should be $\hat{\mathfrak{M}}{ }_{D}$, i.e., if $\mathfrak{G}=(G \times H) /\left(\theta_{G} \times \tilde{\theta}_{H}\right)$ (diagonal $(K \times K))$, then $D \cong L_{1}(\mathfrak{b})$. We shall see to what extent we can establish the truth of

$$
D \cong L_{1}(\mathfrak{5}) \text {. }
$$

We shall assume in what follows that $K$ is compact.

The first step in the process will be the construction of an epimorphism $T: D \rightarrow L_{1}(5)$. It will then be shown that $T$ is an isomorphism if and only if $D$ is semisimple.

We begin by defining two mappings on the functions $f$ of $F_{C}(A, B)$ with finite support:

$$
\begin{aligned}
& T_{1}(c(a \otimes b))(\xi, \eta) \\
& =\int_{K} \int_{K} a\left(\xi-\theta_{G}\left(\zeta_{1}\right)-\theta_{G}\left(\zeta_{2}\right)\right) c\left(\zeta_{1}\right) b\left(\eta-\tilde{\theta}_{H}\left(\zeta_{2}\right)\right) d \zeta_{1} d \zeta_{2}, \\
& T_{2}(c(a \otimes b))(\xi, \eta) \\
& =\int_{K} \int_{K} a\left(\xi-\theta_{G}\left(\zeta_{1}\right)\right) c\left(\zeta_{2}\right) b\left(\eta-\theta_{H}\left(\zeta_{2}\right)-\tilde{\theta}_{H}\left(\zeta_{1}\right)\right) d \zeta_{1} d \zeta_{2} .
\end{aligned}
$$

By changing variables, $\zeta_{2} \rightarrow \zeta_{3}-\zeta_{1}$, we conclude $T_{1}=T_{2} \equiv \tilde{T}$. The usual integration procedures show $\tilde{T}(f) \in L_{1}(\mathfrak{b})$ and that $\tilde{T}$ is bounded. That is, although $\tilde{T}(f)$ is ostensibly a function of $(\xi, \eta)$, actually $\tilde{T}(f)$ is constant on cosets of $Q, \tilde{T}(f) \in L_{1}(\mathfrak{b})$ and for some constant $M,\|\tilde{T}(f)\| \leqq M \gamma_{1}(f)$. Thus $\tilde{T}$ may be extended to all $F_{C}(A, B)$ by continuity. Further manipulation of integrals shows that $\tilde{T}(I)=0$ (since these manipulations are typified in some of the proofs below, they are here omitted). Hence $\tilde{T}$ defines a unique bounded mapping $T: D \rightarrow L_{1}(\mathfrak{G})$.

\section{LEMMA 3. T is multiplicative.}

Proof. Let $\mathfrak{g}$ represent the typical element of $(\mathfrak{G}: \mathfrak{g}=(\xi, \eta) / Q$.

$$
\begin{gathered}
T\left(c_{1} c_{2}\left(a_{1} a_{2} \otimes b_{1} b_{2}\right)\right)(\mathfrak{g}) \\
=\int_{G} \int_{H} \int_{K} \int_{K} \int_{K} a_{1}\left(\xi-\xi_{1}-\theta_{G}\left(\zeta_{1}\right)-\theta_{G}\left(\zeta_{2}\right)\right) a_{2}\left(\xi_{1}\right) c_{1}\left(\zeta_{1}-\zeta_{3}\right) c_{2}\left(\zeta_{3}\right) \\
\cdot b_{1}\left(\eta-\eta_{1}-\tilde{\theta}_{H}\left(\zeta_{2}\right)\right) b_{2}\left(\eta_{1}\right) d \xi_{1} d \eta_{1} d \zeta_{1} d \zeta_{2} d \zeta_{3} .
\end{gathered}
$$

This may be written after the change $\xi_{1} \rightarrow \xi_{1}-\theta_{G}\left(\zeta_{3}\right)$ 


$$
\begin{aligned}
\int_{G} \int_{H}\left[\int_{K} \int_{K} \int_{K} a_{1}\left(\xi-\xi_{1}-\theta_{G}\left(\zeta_{1}\right)-\theta_{G}\left(\zeta_{2}\right)+\theta_{G}\left(\zeta_{3}\right)\right) a_{2}\left(\xi_{1}-\theta_{G}\left(\zeta_{3}\right)\right)\right. \\
\left.\cdot c_{1}\left(\zeta_{1}-\zeta_{3}\right) c_{2}\left(\zeta_{3}\right) b_{1}\left(\eta-\eta_{1}-\tilde{\theta}_{H}\left(\zeta_{2}\right)\right) b_{2}\left(\eta_{1}\right) d \zeta_{1} d \zeta_{2} d \zeta_{3}\right] d \xi_{1} d \eta_{1}
\end{aligned}
$$

and this in turn is

$$
\begin{array}{r}
\int_{\mathfrak{G}}\left\{\int _ { K } \left[\int_{K} \int_{K} \int_{K} a_{1}\left(\xi-\xi_{1}-\theta_{G}\left(\zeta_{1}\right)-\theta_{G}\left(\zeta_{2}\right)+\theta_{G}\left(\zeta_{3}\right)-\theta_{G}\left(\zeta_{4}\right)\right)\right.\right. \\
. a_{2}\left(\xi_{1}-\theta_{G}\left(\zeta_{3}\right)-\theta_{G}\left(\zeta_{4}\right)\right) c_{1}\left(\zeta_{1}-\zeta_{3}\right) c_{2}\left(\zeta_{3}\right) b_{1}\left(\eta-\eta_{1}-\tilde{\theta}_{H}\left(\zeta_{2}\right)-\tilde{\theta}_{H}\left(\zeta_{4}\right)\right) \\
\left.\left.\cdot b_{2}\left(\eta_{1}-\tilde{\theta}_{H}\left(\zeta_{4}\right)\right) d \zeta_{1} d \zeta_{2} d \zeta_{3}\right] d \zeta_{4}\right\} d \mathfrak{g}
\end{array}
$$

We have used the formula

$$
\int_{Q} \Phi(\omega) d \omega=\int_{\text {diagonal }(K \times K)} \Phi\left(\left(\theta_{G} \times \tilde{\theta}_{H}\right)(\zeta, \zeta)\right) d(\zeta, \zeta)=\int_{K} \Phi\left(\left(\theta_{G} \times \tilde{\theta}_{H}\right)(\zeta, \zeta)\right) d \zeta,
$$

valid (if the Haar measures are adjusted) for any $\Phi$ continuous and with compact support. The formula follows from the simple observation: If $\theta: X \rightarrow Y$ is an epimorphism of compact groups, then

$$
\int_{X} \Phi(\theta(x)) d x
$$

is an invariant functional on $L_{1}(Y)$. Thus for some Haar measure on $Y$

$$
\int_{X} \Phi\left(\theta(x) d x=\int_{Y} \Phi(y) d y .\right.
$$

We assume the adjustment has been made. We find then

$$
\begin{aligned}
& T\left(c_{1}\left(a_{1} \otimes b_{1}\right)\right) T\left(c_{2}\left(a_{2} \otimes b_{2}\right)\right)(\xi, \eta) \\
&=\int_{G}\left\{\left[\int_{K} \int_{K} a_{1}\left(\xi-\xi_{1}-\theta_{G}\left(\zeta_{1}\right)-\theta_{G}\left(\zeta_{2}\right)\right) c_{1}\left(\zeta_{1}\right) b_{1}\left(\eta-\eta_{1}-\tilde{\theta}_{H}\left(\zeta_{2}\right)\right) d \zeta_{1} d \zeta_{2}\right]\right. \\
&\left.\cdot\left[\int_{K} \int_{K} a_{2}\left(\xi_{1}-\theta_{G}\left(\zeta_{3}\right)-\theta_{G}\left(\zeta_{4}\right)\right) c_{2}\left(\zeta_{3}\right) b_{2}\left(\eta_{1}-\tilde{\theta}_{H}\left(\zeta_{4}\right)\right) d \zeta_{3} d \zeta_{4}\right]\right\} d g .
\end{aligned}
$$

Changing variables $\left(\zeta_{1} \rightarrow \zeta_{1}-\zeta_{3}\right)$ in the first [] above we obtain

$$
\begin{aligned}
\int_{G}\left\{\left[\int_{K} \int_{K} a_{1}\left(\xi-\xi_{1}-\theta_{G}\left(\zeta_{1}\right)-\theta_{G}\left(\zeta_{2}\right)+\theta_{G}\left(\zeta_{3}\right)\right) c_{1}\left(\zeta_{1}-\zeta_{3}\right)\right.\right. \\
\left.\cdot b_{1}\left(\eta-\eta_{1}-\tilde{\theta}_{H}\left(\zeta_{2}\right)\right) d \zeta_{1} d \zeta_{2}\right] \\
\left.\cdot\left[\int_{K} \int_{K} a_{2}\left(\xi_{1}-\theta_{G}\left(\zeta_{2}\right)-\theta_{G}\left(\zeta_{4}\right)\right) c_{2}\left(\zeta_{3}\right) b_{2}\left(\eta_{1}-\tilde{\theta}_{H}\left(\zeta_{4}\right)\right) d \zeta_{3} d \zeta_{4}\right]\right\} d g
\end{aligned}
$$


We may replace $(\xi, \eta)$ by $(\xi, \eta)-\left(\theta_{G}\left(\zeta_{4}\right), \tilde{\theta}_{H}\left(\zeta_{4}\right)\right)$ in the first [ ] above and obtain

$$
\begin{aligned}
\int_{\mathfrak{G}}\left\{\left[\int_{K} \int_{K} a_{1}\left(\xi-\xi_{1}-\theta_{G}\left(\zeta_{1}\right)-\theta_{G}\left(\zeta_{2}\right)+\theta_{G}\left(\zeta_{3}\right)-\theta_{G}\left(\zeta_{4}\right)\right) c_{1}\left(\zeta_{1}-\zeta_{3}\right)\right.\right. \\
\left.\cdot b_{1}\left(\eta-\eta_{1}-\tilde{\theta}_{H}\left(\zeta_{2}\right)-\tilde{\theta}_{H}\left(\zeta_{4}\right)\right) d \zeta_{1} d \zeta_{2}\right] \\
\left.\cdot\left[\int_{K} \int_{K} a_{2}\left(\xi_{1}-\theta_{G}\left(\zeta_{3}\right)-\theta_{G}\left(\zeta_{4}\right)\right) c_{2}\left(\zeta_{3}\right) b_{2}\left(\eta_{1}-\tilde{\theta}_{H}\left(\zeta_{4}\right)\right) d \zeta_{3} d \zeta_{4}\right]\right\} d \mathfrak{g} .
\end{aligned}
$$

Comparison of the formulas for $T\left(c_{1} c_{2}\left(a_{1} a_{2} \otimes b_{1} b_{2}\right)\right)$ and $T\left(c_{1}\left(a_{1} \otimes b_{1}\right)\right)$ - $T\left(c_{2}\left(a_{2} \otimes b_{2}\right)\right)$ shows they are the same and we conclude that $T$ is multiplicative.

LEMMA 4. Let $\tilde{A}, \tilde{C}$ be commutative Banach algebras, $\tilde{A}$ a $\tilde{C}$-bimodule. Let $\{\tilde{c}\}$ be an approximate identity for $\tilde{C}$. Then $\{\tilde{c}\}$ is also an approximate identity for $\tilde{A}$ if and only if each $\tilde{a}_{1} \in \tilde{A}$ is of the form $\tilde{c}_{1} \tilde{a}_{2}, \tilde{c}_{1} \in \tilde{C}, \tilde{a}_{2} \in \tilde{A}$.

Proof. Paraphrasing Cohen's argument [3], we find that if $\{\tilde{c}\}$ is an approximate identity for $\tilde{A}$, then each $\tilde{a}_{1} \in \tilde{A}$ is of the form indicated. Conversely if $\tilde{a}_{1} \in \tilde{A}$, let $\tilde{a}_{1}=\tilde{c}_{1} \tilde{a}_{2}$. Then $\tilde{c} \tilde{a}_{1}-\tilde{a}_{1}=\tilde{c} \tilde{c}_{1} \tilde{a}_{2}-\tilde{c}_{1} \tilde{a}_{2}=\left(\tilde{c} \tilde{c}_{1}-\tilde{c}_{1}\right) \tilde{a}_{2} \rightarrow 0$ on the net $\{\tilde{c}\}$.

LEMMA 5. Let $K$ be compact and let $\{c\}$, where all $c$ are continuous and nonnegative, be an approximate identity in $C=L_{1}(K)$. Then for $a \in A=L_{1}(G)$,

on the net $\{c\}$.

$$
a_{c}(\xi)=\int_{K} a\left(\xi-\theta_{G}(\zeta)\right) c(\zeta) d \zeta \rightarrow a
$$

Proof. We may assume that $a$ is continuous, nonnegative and that $a$ has compact support. Hence there is a fixed compact $\Lambda \subset G$ such that $a$ and all $a_{c}$ are 0 off $\Lambda$. Then we write

$$
\begin{aligned}
\int_{G}\left|a(\xi)-a_{c}(\xi)\right| & d \xi \\
= & M_{1} \int_{G / \theta_{G}(K)}\left(\int_{K}\left|a\left(\xi+\theta_{G}(\zeta)\right)-a_{c}\left(\xi+\theta_{G}(\zeta)\right)\right| d \zeta\right) d \lambda
\end{aligned}
$$

where $\lambda=\xi / \theta_{G}(K)$ and where $M_{1}$ is some constant. (An adjustment of Haar measure would make $M_{1}=1$. This would have to be reconciled with the earlier adjustment used in the proof of Lemma 3 . For our purposes, the above suffices.) Our assumptions imply that the functions

$$
\int_{K} a\left(\xi+\theta_{G}(\zeta)\right) d \zeta, \quad \int_{K} a_{c}\left(\xi+\theta_{G}(\zeta)\right) d \zeta,
$$

in $L_{1}\left(G / \theta_{G}(K)\right)$ are continuous and that they all vanish off the compact set $\Lambda / \theta_{G}(K)$.

For $\varepsilon>0$, and each $\xi+\theta_{G}(K) \in \Lambda / \theta_{G}(K)$, choose $\delta_{1}>0$ and $c_{\xi}$ so that 


$$
\int_{\mathbf{K}}\left|a\left(\xi+\theta_{G}(\zeta)\right)-a_{c}\left(\xi+\theta_{G}(\zeta)\right)\right| d \zeta<\delta_{1}
$$

for $c_{\xi}$ and all $c$ beyond $c_{\xi}$. Let $\delta_{2}>0$ and let $V$ be a neighborhood of 0 in $G / \theta_{G}(K)$ such that for $\xi_{1}+\theta_{G}(K) \epsilon \xi+\theta_{G}(K)+V, \int_{K}\left|a\left(\xi+\theta_{G}(\zeta)\right)-a\left(\xi_{1}+\theta_{G}(\zeta)\right)\right| d \zeta<\delta_{2}$. Then, for such $\xi, \xi_{1}$,

$$
\begin{aligned}
\mid \int_{K}\left(a_{c}(\xi+\right. & \left.\theta_{G}(\zeta)\right)-a_{c}\left(\xi_{1}+\theta_{G}(\zeta)\right) d \zeta \mid \\
& =\left|\int_{K} \int_{K}\left(a\left(\xi+\theta_{G}(\zeta)-\theta_{G}\left(\zeta_{1}\right)\right)-a\left(\zeta_{1}+\theta_{G}(\zeta)-\theta_{G}\left(\zeta_{1}\right)\right)\right) c\left(\zeta_{1}\right) d \zeta d \zeta_{1}\right| \\
& \leqq \int_{K}\left|c\left(\zeta_{1}\right)\right|\left(\int_{K}\left|a\left(\xi+\theta_{G}(\zeta)\right)-a\left(\xi_{1}+\theta_{G}(\zeta)\right)\right| d \zeta\right) d \zeta_{1} \\
& <\delta_{2} .
\end{aligned}
$$

In other words, the functions $\int_{K} a\left(\xi+\theta_{G}(\zeta)\right) d \zeta, \int_{K} a_{c}\left(\xi+\theta_{G}(\zeta)\right) d \zeta$ form an equicontinuous family. Hence for each $\xi+\theta_{G}(K) \in \Lambda / \theta_{G}(K)$ there is a neighborhood $V\left(\xi+\theta_{G}(K)\right)$ where the inequality $\left({ }^{*}\right)$ persists for all $c$ beyond $c_{\xi}$. Let $V\left(\xi_{i}+\theta_{G}(K)\right), i=1,2, \cdots, N$ be a finite subcovering of $\Lambda / \theta_{G}(K)$. Then for all $c$ beyond the "last" $c_{\xi_{i}}$ and for any $\xi+\theta_{G}(K) \in \Lambda / \theta_{G}(K),\left({ }^{*}\right)$ is true. Hence if

$$
\delta_{1} M_{1} \int_{\Lambda / \theta_{G}(K)} d \lambda<\varepsilon,
$$

we see $\int_{G}\left|a(\xi)-a_{c}(\xi)\right| d \xi<\varepsilon$ for all $c$ beyond the "last" $c_{\xi i}$, i.e.,

$$
\lim _{\{c\}} \int_{G}\left|a(\xi)-a_{c}(\xi)\right| d \xi=0 .
$$

Furthermore, since $\|c\| \equiv 1$, we find $\left\|a_{c}\right\| \leqq\|a\|$.

Combining Lemmas 4 and 5 we see that there is an approximate identity $\{c\}$ for $C$ that is also an approximate identity for $A$, and mutatis mutandis for $B$.

LEMMa 6. $T$ ' is surjective.

Proof. Let $\phi \in L_{1}(\mathfrak{5})$. If $R: L_{1}(G \times H) \rightarrow L_{1}(\mathfrak{G})$ is the canonical epimorphism

$$
R(\psi)=\int_{Q} \psi((\xi, \eta)+q) d q,
$$

let $R(\Phi)=\phi$. Via [7, p. 59-60], [9] we can find a $\tilde{z} \in A \otimes_{\gamma} B, \tilde{z}$ represented by

$$
\sum_{n=1}^{\infty}\left(\tilde{a}_{n} \otimes b_{n}\right) \text {, }
$$

such that in the isomorphism $A \otimes_{\gamma} B \leftrightarrow L_{1}(G \times H), \tilde{z} \leftrightarrow \Phi$. Exploiting Lemmas 
4 and 5 we find elements $c_{n} \in C$ and $a_{n} \in A$ such that $\tilde{a}_{n}=a_{n} c_{n}$. It is now clear that if $z$ in $D$ is represented by

$$
\sum_{n=1}^{\infty} c_{n}\left(a_{n} \otimes b_{n}\right)
$$

then $T(z)=\phi$.

THEOREM 1. T is an isomorphism if and only if $D$ is semisimple.

Since $\left(\mathfrak{b}\right.$ is a locally compact abelian group, $L_{1}(\mathfrak{G})$ is semisimple and thus if $T$ is an isomorphism, $D$ is semisimple.

On the other hand, let $D$ be semisimple. We shall show that for any $z \in T^{-1}(0), \hat{z}\left(M_{D}\right) \equiv 0$, whence $z=0, T$ is $1-1$ and, from the earlier results, an isomorphism. To this end let

$$
\sum_{n=1}^{\infty} c_{n}\left(a_{n} \otimes b_{n}\right)
$$

represent $z \in T^{-1}(0)$. For any $M_{D}$ let $\tau\left(M_{D}\right)=(\alpha, \beta)$ where $\theta_{G}^{*}(\alpha)=\theta_{H}^{*}(\beta)=\gamma \in \hat{K}$. Then

$$
\begin{aligned}
& \hat{z}\left(M_{D}\right)=\sum_{n=1}^{\infty} \hat{c}_{n}(\gamma) \hat{a}_{n}(\alpha) \hat{b}_{n}(\beta) \\
&=\sum_{n=1}^{\infty} \int_{G} \int_{H}\left(\int_{K} a_{n}\left(\xi-\theta_{G}\left(\zeta_{i}\right)\right) c_{n}\left(\zeta_{1}\right) b_{n}(\eta) d \zeta_{1}\right) \overline{(\xi, \alpha)(\eta, \beta)} d \xi d \eta \\
&=\sum_{n=1}^{\infty} \int_{\mathfrak{G}}\left\{\int_{K}\left[\int_{K} a_{n}\left(\xi+\theta_{G}\left(\zeta_{2}\right)-\theta_{G}\left(\zeta_{1}\right)\right) c_{n}\left(\zeta_{1}\right) b_{n}\left(\eta+\tilde{\theta}_{H}\left(\zeta_{2}\right)\right) d \zeta_{1}\right]\right. \\
&\left.\cdot \overline{\left(\xi+\theta_{G}\left(\zeta_{2}\right), \alpha\right)} \frac{\left(\eta+\tilde{\theta}_{H}\left(\zeta_{2}\right), \beta\right)}{(\bar{l})} d \zeta_{2}\right\} d \mathfrak{g}
\end{aligned}
$$

where $\mathfrak{g}$ denotes the coset of $(\xi, \eta)$ in $\mathfrak{G}$,

$$
\left(\xi+\theta_{G}\left(\zeta_{2}\right), \alpha\right)\left(\eta+\hat{\theta}_{H}\left(\zeta_{2}\right), \beta\right)=(\mathfrak{g},\{\alpha, \beta\})
$$

where $\{\alpha, \beta\}$ is the element of $\hat{\mathfrak{G}}$ corresponding to the pair $(\alpha, \beta) \in Q^{\perp}$. Setting $T\left(c_{n}\left(a_{n} \otimes b_{n}\right)\right)=\psi_{n}(\mathrm{~g})$ we find the above sum of integrals reduces to

$$
\sum_{n=1}^{\infty} \int_{\mathfrak{G}} \psi_{n}(\mathfrak{g})(\mathfrak{g},\{\alpha, \beta\}) d \mathfrak{g}=[(T z)]^{\wedge}\{\alpha, \beta\} \equiv 0 .
$$

Hence $z=0$ and $T$ is an isomorphism.

Note that when $C=C, D$ is semisimple, $\mathfrak{G}=G \times H$ and a known result [5], [9] obtains: $L_{1}(G) \otimes_{\gamma} L_{1}(H)=L_{1}(G \times H)$.

In [6] the relationship between Grothendieck's "condition of approximation" and its equivalents [7] on the one hand and the semisimplicity of $A \otimes_{\gamma} B$, when $A$ and $B$ are semisimple, on the other hand were examined. 
Pursuit of the same line here does not appear promising. The method that succeeds in the case of $A \otimes_{\gamma} B$ would require, in the context of $L_{1}(G) \otimes_{L_{1}(K)} L_{1}(H)$, the presence of many finite-dimensional $L_{1}(K)$-modules in $L_{1}(G)$. However, if $G=R, K=Z$ and if $\theta_{G}$ is the inclusion map, then $L_{1}(G)$ has no finite-dimensional $L_{1}(K)$-modules other than $\{0\}$. This fact can be verified quite easily as follows:

Let $N$ be a nontrivial finite-dimensional $L_{1}(Z)$-module in $L_{1}(R)$ and let $\left\{a_{i}\right\}_{i=1}^{n}$ be a basis for $N$. For some integer $r$, real numbers $t_{1}, t_{2}, t_{3}, t_{4}$, and some $c$ in $L_{1}(Z)$ the following hold:

(a) $r<t_{1}<t_{2}<t_{3}<t_{4}<r+1$;

(b) $\hat{c}(\tilde{t})=0$ if $0<\tilde{t}_{1}<\tilde{t}<\tilde{t}_{2}<1, \hat{c}(\tilde{t})=1$ if $0<\tilde{t}_{3}<\tilde{t}<\tilde{t}_{4}<1$, where $\tilde{t}=\boldsymbol{t}(\bmod 1)$

(c) $\left\{\hat{a}_{i}(\tilde{t})\right\}$ are linearly independent on $\left(\tilde{t}_{1}, \tilde{t}_{2}\right)$ and on $\left(\tilde{t}_{3}, \tilde{t}_{4}\right)$.

If $c a_{i}=\sum_{j=1}^{n} \lambda_{i j} a_{j}$ we rapidly conclude the contradiction: $\lambda_{i j}=\delta_{i j}=0$ for all $i, j=1,2, \cdots, n$.

4. Tensor products of groups. In the setting most briefly described by the symbols $\left\{G, H, K, \theta_{G}, \theta_{H}\right\}$ we may interpret $K$ as operating on each of $G$ and $H$ according to the following format: Let $G=\{\xi\}, H=\{\eta\}, K=\{\zeta\}$. Define the action of $\zeta \in K$ on $\xi \in G$ by $\zeta(\xi)=\xi+\theta_{G}(\zeta)$. Similarly $\zeta(\eta)=\eta+\theta_{H}(\zeta)$. Thus each $\zeta \in K$ induces 1-1 mappings of $G$ and $H$ upon themselves.

It is simple to give abstract characterizations of the actions of these mappings. Then, in $G \times H$ we may introduce a relation $S$ by the rule: $\left(\xi_{1}, \eta_{1}\right) S\left(\xi_{2}, \eta_{2}\right)$ if and only if there is a $\zeta \in K$ such that $\zeta\left(\xi_{1}\right)=\xi_{2}$ and $\zeta\left(\eta_{2}\right)=\eta_{1}$. The relation $S$ is easily shown to be an equivalence relation because $K$ is abelian. The $S$-equivalence class containing $\left(0_{G}, 0_{H}\right)$, the identity of $G \times H$, is then readily seen to be a subgroup (normal since $G$ and $H$ are abelian) which we may readily identify with what we have already denoted by $Q$. In terms of the mappings that $K$ induces on $G$ and $H$, we may then recover $\theta_{G}$ and $\theta_{H}$ by the formulas $\theta_{G}(\zeta)=\zeta\left(0_{G}\right), \theta_{H}(\zeta)=\zeta\left(0_{H}\right)$.

In the light of the above we choose to interpret $G \times H / Q$ as the tensor product $G \otimes_{K} H$ relative to the mappings $\theta_{G}$ and $\theta_{H}$ or (equivalently) relative to the defined actions of $K$ on $G$ and $H$.

If we show then that $L_{1}(G) \otimes_{L_{1}(K)} L_{1}(H)$ and $L_{1}((5)$ are isomorphic we shall simultaneously show that the tensor product of group algebras is the group algebra of the tensor product.

The theorem we shall prove is

THEOREM 1. If $G$ and $H$ are locally compact abelian groups and if $K$ is $a$ compact abelian group, then

$$
L_{1}(G) \otimes_{L_{1}(K)} L_{1}(H) \cong L_{1}\left(G \otimes_{K} H\right)
$$

where the tensor products in question are taken with respect to continuous homomorphisms $\theta_{G}$ and $\theta_{H}$ of $K$ into $G$ and $H$. 
This result was proved in 1962. During an unavoidable delay in the publication process, Boaz Natzitz had the opportunity to work with the author and he extended the theorem to the case where $K$ is permitted to be locally compact (and abelian). His efforts led to a doctoral dissertation at the University of Minnesota, and his paper (incorporating new methods) will be published at a later date.

We begin the proof of the theorem with

Lemma 1. Let $G$ be a locally compact abelian group and let $a \in L_{1}(G)$. Then for $\varepsilon>0$ there is a $u \in L_{1}(G)$ such that $\|u\| \leqq 2, u$ is continuous, $u$ has compact support and such that $\|a-a u\|<\varepsilon$.

Proof. Let $v \in L_{1}(G)$ be such that $\|a-a v\|<\varepsilon / 2,\|v\|=1$. Choose $u$ continuous in $L_{1}(G)$ and so that $\|u\| \leqq 2,\|v-u\|<\varepsilon / 2\|a\|$ (to avoid the trivial case we assume $a \neq 0$ ) and $u$ has compact support [10]. Then $\|a-a u\|$ $\leqq\|a-a v\|+\|a v-a u\|<\varepsilon / 2+\varepsilon / 2=\varepsilon$.

Now let $\tilde{c}_{y}=(\zeta, \gamma)$ where $\zeta \in K, \gamma \in \hat{K}$. Then $\tilde{c}_{\gamma} \in L_{1}(K)$. If $a \in L_{1}(G)$ and if $\hat{a}$ has compact support then

$$
\begin{aligned}
\tilde{c}_{\gamma} a & =\int_{K} a\left(\xi-\theta_{G}(\zeta)\right)(\zeta, \gamma) d \zeta \\
& =\int_{K}\left(\int_{\widehat{G}} \hat{a}(\alpha)\left(\xi-\theta_{G}(\zeta), \alpha\right) d \alpha\right)(\zeta, \gamma) d \zeta .
\end{aligned}
$$

Hence, the fundamental relations among group characters of compact groups show

$$
\begin{aligned}
\tilde{c}_{\gamma} a & =\int_{\widehat{G}} \hat{a}(\alpha)(\xi, \alpha)\left(\int_{K}\left(\zeta, \gamma-\theta_{G}^{*}(\alpha)\right) d \zeta\right) d \alpha \\
& =\int_{\theta_{G}^{*-1}(\gamma)} \hat{a}(\alpha)(\xi, \alpha) d \alpha .
\end{aligned}
$$

Let $z^{\prime \prime} \in D$ be represented by $\sum_{n=1}^{\infty} c_{n}^{\prime \prime}\left(a_{n}^{\prime \prime} \otimes b_{n}^{\prime \prime}\right)$ and be such that $\hat{z}^{\prime \prime}\left(M_{D}\right) \equiv 0$. By the use of approximate identities (cf. Lemma 1) we can for $\varepsilon>0$ produce a $z^{\prime}$ represented by $\sum_{n=1}^{\infty} c_{n}\left(a_{n}^{\prime} \otimes b_{n}^{\prime}\right)$ such that $\gamma\left(z^{\prime}-z^{\prime \prime}\right)<\varepsilon / 2, \hat{z}^{\prime}\left(M_{D}\right) \equiv 0$, and such that all $\hat{a}_{n}^{\prime}, \hat{b}_{n}^{\prime}, \hat{c}_{n}$ have compact support. Actually, and we shall need this in the course of our argument, there are fixed compact sets $\Gamma, \mathrm{H}, \mathrm{K}$ in $\hat{G}, \hat{H}, \hat{K}$ such that for all $n$, support $\left(\hat{a}_{n}^{\prime}\right) \subset \Gamma$, support $\left(\hat{b}_{n}^{\prime}\right) \subset \mathrm{H}$, support $\left(\hat{c}_{n}\right) \subset \mathrm{K}$. Finally, by the use of two functions $u, v$, nonnegative and with compact supports, $C_{u}, D_{v}$ we may write $a_{n}=u a_{n}^{\prime}, b_{n}=v b_{n}^{\prime}$ and conclude that $\sum_{n=1}^{\infty} c_{n}\left(a_{n} \otimes b_{n}\right)$ represents an element $z$ such that $\gamma\left(z^{\prime}-z\right)<\varepsilon / 2^{2}, \hat{z}\left(M_{D}\right) \equiv 0$. The exploitation of $\left(3^{\prime}\right),\left(4^{\prime}\right), \S 1$, permits us to assume $\sum_{n=1}^{\infty}\left\|a_{n}^{\prime}\right\|, \sum_{n=1}^{\infty}\left\|b_{n}^{\prime}\right\|<\infty$.

Since $K$ is compact, $\hat{K}$ is discrete, $\mathrm{K}$ is finite and each $c_{n}=\sum_{\gamma \in \kappa} \hat{c}_{n}(\gamma)(\zeta, \gamma)$. We conclude that 


$$
\begin{aligned}
\sum_{n=1}^{\infty}\left(\sum_{\gamma \in \kappa} \hat{c}_{n}(\gamma) \hat{c}_{\gamma}\right) \\
\quad \cdot\left(\int_{G} \int_{\hat{G}} \hat{a}_{n}^{\prime}(\alpha)\left(\xi_{1}, \alpha\right) u\left(\xi-\xi_{1}\right) d \xi_{1} d \alpha \otimes \int_{H} \int_{\hat{H}} \hat{b}_{n}^{\prime}(\beta)\left(\eta_{1}, \beta\right) v\left(\eta-\eta_{1}\right) d \eta_{1} d \beta\right)
\end{aligned}
$$

represents $z$.

Next we write for each $\gamma \in \mathbf{K}$,

$$
\begin{aligned}
& \int_{\hat{G}}=\int_{\theta_{G}^{*-1}(\gamma)}+\backslash \int_{\widehat{G} \mid \theta_{G}^{*-1}(\gamma)}, \\
& \int_{\hat{H}}=\int_{\theta_{H}^{*-1}(\gamma)}+\backslash \int_{\hat{H} \mid \theta_{H}^{*-1}(\gamma)} .
\end{aligned}
$$

From Lemma $1, \S 1$ we see that if $c c^{\prime}(a \otimes b)$ represents some $w$ and $c^{\prime} a=0$, then $w=0$. In the present circunstances, if $c(a \otimes b)$ represents some $w$ and $c a=0$, then $w=0$. For if $c^{\prime}$ is properly chosen so that $\left\|c^{\prime} c-c\right\|$ is small, then $c c^{\prime}(a \otimes b)$ represents a $w^{\prime}$ near $w$ and on the other hand $w^{\prime}=0$.

From the observations

$$
\tilde{c}_{\gamma} a=\int_{\theta_{G}^{*-1}(\gamma)} \hat{a}(\alpha)(\xi, \alpha) d \alpha
$$

and $\tilde{c}_{\gamma} * \tilde{c}_{\gamma}=\tilde{c}_{\gamma}$ we conclude that $z$ is represented by

$$
\begin{aligned}
& \sum_{n=1}^{\infty}\left(\sum_{\gamma \in \mathbf{K}} \tilde{c}_{n}(\gamma) \tilde{c}_{\gamma}\right) \\
& \cdot\left(\int_{G} \int_{\theta_{G}^{*-1}(\gamma)} \hat{a}_{n}^{\prime}(\alpha)\left(\xi_{1}, \alpha\right) u\left(\xi-\xi_{1}\right) d \xi_{1} d \alpha \otimes \int_{H} \int_{\theta_{H}^{*-1}(\gamma)} \hat{b}_{n}^{\prime}(\beta)\left(\eta_{1}, \beta\right) v\left(\eta-\eta_{1}\right) d \eta_{1} d \beta\right) .
\end{aligned}
$$

If $z_{N}$ is represented by the first $N$ terms of the above series, then $\gamma\left(z_{N}-z\right) \rightarrow 0$ as $N \rightarrow \infty$. We shall show that for each $N$, there are finitely many precompact disjoint measurable sets $C_{i}, D_{j}$ in $G, H$, elements $\tilde{\xi}_{i} \in C_{i}, \tilde{\eta}_{j} \in D_{j}$ for which $\sum_{i} m_{G}\left(C_{i}\right)$, $\sum_{j} m_{H}\left(D_{j}\right)<T_{0}<\infty$, where $T_{0}$ is $N$-free, and for which

$$
\begin{array}{r}
\sum_{\gamma \in \mathbf{K}}\left(\sum_{i, j}\left(\int_{\theta_{G}^{*-1}(\gamma)} \int_{\theta_{H}^{*-1}(\gamma)} \sum_{n=1}^{N} \hat{c}_{n}(\gamma) \hat{a}_{r}^{\prime}(\alpha) \hat{b}_{n}^{\prime}(\beta)\left(\tilde{\xi}_{i}, \alpha\right)\left(\tilde{\eta}_{j}, \beta\right) d \alpha d \beta\right)\right. \\
\left.\cdot\left(m_{G}\left(C_{i}\right) u\left(\xi-\tilde{\xi}_{i}\right) \otimes m_{H}\left(D_{j}\right) v\left(\eta-\tilde{\eta}_{j}\right)\right)\right)
\end{array}
$$

represents a $\tilde{z}_{N}$ such that $\gamma\left(\tilde{z}_{N}-z_{N}\right)<\varepsilon / 2^{4}$, for all $N$. Assuming this we finish the proof as follows: First we see $\sum_{n=1}^{\infty} \hat{c}_{n}(\gamma) \hat{a}_{n}^{\prime}(\alpha) \hat{b}_{n}^{\prime}(\beta)=0$ in the ranges of integration, and $S=\sum_{n=1}^{\infty}\left\|c_{n}\right\|\left\|a_{n}^{\prime}\right\|\left\|b_{n}^{\prime}\right\|<\infty$. Thus for some $N_{0}$ depending only on $S$ and on the measure of $H \times \mathrm{K}$

$$
\left\|\left(\int_{\theta_{G}^{*-1}(\gamma)} \int_{\theta_{H}^{*-1}(\gamma)} \sum_{n=1}^{N} \hat{c}_{n}(\gamma) \hat{a}_{n}^{\prime}(\alpha) \hat{b}_{n}^{\prime}(\beta)\left(\tilde{\xi}_{i}, \alpha\right)\left(\tilde{\eta}_{j}, \beta\right) d \alpha d \beta\right) \tilde{c}_{\gamma}\right\|<\delta
$$


where $\delta \cdot m_{\mathrm{K}}(\mathrm{K}) \cdot T_{0}^{2}<\varepsilon / 2^{5}$, if $N \geqq N_{0}$. If $r_{i}=m_{G}\left(C_{i}\right), s_{j}=m_{K}\left(D_{j}\right)$, then

$$
\begin{aligned}
\gamma\left(\tilde{z}_{N}\right) & <\delta \cdot m_{K}(\mathrm{~K})\left(\sum_{i, j} r_{i}\left\|u\left(\xi-\tilde{\xi}_{i}\right)\right\| s_{j}\left\|v\left(\eta-\tilde{\eta}_{j}\right)\right\|\right) \\
& =\delta \cdot m_{K}(\mathrm{~K}) \sum_{i, j} r_{i} s_{j}=\delta \cdot m_{K}(\mathrm{~K})\left(\sum_{i} r_{i}\right)\left(\sum_{j} s_{j}\right) \\
& <\delta \cdot m_{K}(\mathrm{~K}) T_{0}^{2}<\varepsilon / 2^{5} .
\end{aligned}
$$

Hence $\gamma\left(\tilde{z}_{N}\right)<\varepsilon / 2^{5}$.

In summary, we choose $N_{0}$ so that (**) holds for $N \geqq N_{0}$, then $N>N_{0}$, so that $\gamma\left(z_{N}-z\right)<\varepsilon / 2^{3}$, then $C_{i}, D_{j}, \xi_{i}, \eta_{j}$ so that $\gamma\left(\tilde{z}_{N}-z_{N}\right)<\varepsilon / 2^{4}$. As above, $\gamma\left(\tilde{z}_{N}\right)<\varepsilon / 2^{5}$ and

$$
\gamma\left(z^{\prime \prime}\right) \leqq \gamma\left(z^{\prime \prime}-z^{\prime}\right)+\gamma\left(z^{\prime}-z\right)+\gamma\left(z-z_{N}\right)+\gamma\left(z_{N}-\tilde{z}_{N}\right)+\gamma\left(\tilde{z}_{N}\right)<\varepsilon .
$$

Since $\varepsilon>0$ is arbitrary, we see $z^{\prime \prime}=0$.

Thus we must prove the statement concerning the $r_{i}, s_{j}, \cdots$.

For brevity we set $\tilde{c}_{j} a_{n}^{\prime}=d_{n \gamma}, \tilde{c}_{\gamma} b_{n}^{\prime}=e_{n \gamma}$. Then $z_{N}$ is represented by $\sum_{n=1}^{N}\left(\sum_{\gamma \in \mathbf{K}} \hat{c}_{n}(\gamma) \tilde{z}_{j}\right)\left(d_{n \gamma} u \otimes e_{n \gamma} v\right)$. For $\rho>0$ there are compact sets $C_{d}, D_{e}$ such that

$$
\int_{G \backslash C_{d}} \sum_{n=1}^{\infty} \sum_{\gamma \in \mathbf{K}}\left|d_{n \gamma}(\xi)\right| d \xi, \int_{H \backslash D_{e}} \sum_{n=1}^{\infty} \sum_{\gamma \in \mathbf{K}}\left|e_{n \gamma}(\eta)\right| d \eta<\rho ! 2,
$$

since $\sum_{n=1}^{\infty}\left\|a_{n}^{\prime}\right\| \sum_{n=1}^{\infty}\left\|b_{n}^{\prime}\right\|<\infty$. Hence there are compact sets $\bar{C}, \bar{D}$, independent of $N$ such that $w_{N}$ represented by

$$
\sum_{n=1}^{N}\left(\sum_{\gamma \in \mathbf{K}} \hat{c}_{n}(\gamma) \tilde{c}_{\gamma}\right)\left(\int_{\bar{c}} d_{n \gamma}\left(\xi_{1}\right) u\left(\xi-\xi_{1}\right) d \xi_{1} \otimes \int_{\bar{D}} e_{n \gamma}\left(\eta_{1}\right) v\left(\eta-\eta_{1}\right) d \eta_{1}\right)
$$

satisfies $\gamma\left(z_{N}-w_{N}\right)<\rho / 2^{2}$. The functions $d_{n \gamma}, e_{n \gamma}$ are uniformly continuous and thus for $\sigma>0$ there are neighborhoods $X(0), Y(0)$ in $G, H$, such that

$$
\left|d_{n \gamma}\left(\xi_{1}\right)-d_{n \gamma}\left(\xi_{2}\right)\right|,\left|e_{n \gamma}\left(\eta_{1}\right)-e_{n \gamma}\left(\eta_{2}\right)\right|,\left\|u_{\xi_{1}}-u_{\xi_{2}}\right\|,\left\|v_{\eta_{1}}-v_{\eta_{2}}\right\|<\sigma
$$

if $\xi_{1}-\xi_{2} \in X(0), \eta_{1}-\eta_{2} \in Y(0)$.

If we cover $\bar{C}$ and $\bar{D}$ with finitely many translates of $X(0)$ and $Y(0)$ and then partition $\bar{C}$ and $\bar{D}$ into finitely many measurable precompact sets $C_{i}, D_{j}$ each of which is contained in one of the chosen translates of $X(0), Y(0)$, and if $\tilde{\xi}_{i} \in C_{i}, \tilde{\eta}_{j} \in D_{j}$, we can estimate

$$
\begin{aligned}
& \left\|\int_{\bar{C}} d_{n \gamma}\left(\xi_{1}\right) u\left(\xi-\xi_{1}\right) d \xi_{1}-\sum_{i} d_{n \gamma}\left(\tilde{\xi}_{i}\right) u\left(\xi-\tilde{\xi}_{i}\right) m_{G}\left(C_{i}\right)\right\|, \\
& \left\|\int_{\bar{D}} e_{n \gamma}\left(\eta_{1}\right) v\left(\eta-\eta_{1}\right) d \eta_{1}-\sum_{j} e_{n \gamma}\left(\tilde{\eta}_{j}\right) v\left(\eta-\tilde{\eta}_{j}\right) m_{H}\left(D_{j}\right)\right\| .
\end{aligned}
$$


Indeed (we estimate only one of the above, the other is treated similarly),

$$
\begin{aligned}
\left\|\int_{\bar{c}_{i}}-\sum_{i}\right\| & =\left\|\sum_{i} \int_{C_{i}}-\sum_{i}\right\| \\
& \leqq\left\|\sum_{i} \int_{C i} d_{n \gamma}\left(\xi_{1}\right)\left(u\left(\xi-\xi_{1}\right)-u\left(\xi-\tilde{\xi}_{i}\right)\right) d \xi_{1}\right\|+\sigma m_{G}\left(\bar{C}_{i}\right)\|u\| \\
& \leqq \sum_{i} \int_{c_{i}}\left|d_{n \gamma}\left(\xi_{1}\right)\right|\left(\int_{G}\left|u\left(\xi-\xi_{1}\right)-u\left(\xi-\tilde{\xi}_{1}\right)\right| d \xi\right) d \xi_{1}+\sigma m_{G}(\bar{C}) \\
& \leqq \sigma\left(\int_{n \gamma}\left|d_{n \gamma}\left(\xi_{1}\right)\right| d \xi_{1}+m_{G}(\bar{C})\right) .
\end{aligned}
$$

It is now clear that for $\sigma>0$ small enough, $\tilde{w}_{N}$ represented by

$$
\sum_{n=1}^{N}\left(\sum_{\gamma \in \mathbf{K}} \hat{c}_{n}(\gamma) \tilde{c}_{\gamma}\right)\left(\sum_{i} d_{n \gamma}\left(\tilde{\xi}_{j}\right) u\left(\xi-\tilde{\xi}_{i}\right) m_{G}\left(C_{i}\right) \otimes \sum_{j} e_{n \gamma}\left(\tilde{\eta}_{j}\right) v\left(\eta-\tilde{\eta}_{j}\right) m_{H}\left(D_{j}\right)\right)
$$

satisfies $\gamma\left(\tilde{w}_{N}-w_{N}\right)<\rho / 2^{2}$. It is clear that

$$
\begin{array}{r}
\sum_{\gamma \in \mathbf{K}} \sum_{i, j}\left(\int_{\theta_{G}^{*-1}(\gamma)} \int_{\theta_{H}^{*-1}(\gamma)} \sum_{n=1}^{N} \hat{c}_{n}(\delta) \hat{a}_{n}^{\prime}(\alpha) \hat{b}_{n}^{\prime}(\beta)\left(\tilde{\xi}_{i}, \alpha\right)\left(\tilde{\eta}_{j}, \beta\right) d \alpha d \beta \tilde{c}_{\gamma}\right. \\
\cdot\left(m_{G}\left(C_{i}\right) u\left(\xi-\tilde{\xi}_{j}\right) \otimes m_{H}\left(D_{j}\right) v\left(\eta-\tilde{\eta}_{j}\right)\right) .
\end{array}
$$

represents $\tilde{w}_{N}$, and that $\sum_{i} m_{G}\left(C_{i}\right), \sum_{j} m_{H}\left(D_{j}\right) \leqq \max \left(m_{G}(\bar{C}), m_{H}(\bar{D})\right)=T_{0}<\infty$. Hence for small enough $\rho, \gamma\left(\tilde{z}_{N}-z_{N}\right)<\rho / 2+\rho / 2^{2}<\varepsilon / 2^{4}$. The proof is over.

In the theory of group extensions [2] two fixed groups $K, S$ are considered and the exact sequences

$$
0 \rightarrow K \stackrel{\theta_{G}}{\longrightarrow} G \stackrel{\phi_{G}}{\longrightarrow} S \rightarrow 0
$$

are gathered into equivalence classes. Two such sequences (one with $G$, one with $H$ ) are considered equivalent if and only if there is an isomorphism $\psi_{G H}: G \rightarrow H$ such that

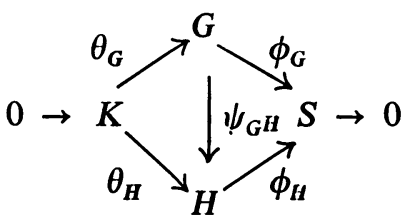

Is a commutative diagram. If $G$ and $H$ are class representatives (the diagram may or may not be commutative), the group

$$
J=\left[\left(\phi_{G} \times \phi_{H}\right)^{-1}(\operatorname{diagonal}(S \times S))\right] /\left[\left(\theta_{G} \times \tilde{\theta}_{H}\right)(\operatorname{diagonal}(K \times K))\right]
$$

is again associated with an exact sequence 


$$
0 \rightarrow K \stackrel{\theta_{J}}{\longrightarrow} J \stackrel{\phi_{J}}{\longrightarrow} S \rightarrow 0
$$

representative of the Baer product [1], [2] of the originally given classes.

In our discussion

$$
\mathfrak{G}=G \times H /\left[\left(\theta_{G} \times \tilde{\theta}_{H}\right)(\operatorname{diagonal}(K \times K))\right]
$$

and

$$
\hat{\mathfrak{G}}=\left[\left(\theta_{G}^{*} \times \theta_{H}^{*}\right)^{-1}(\operatorname{diagonal}(\hat{K} \times \hat{K}))\right]^{\perp} .
$$

In particular $\mathfrak{G}$ is a supergroup of a representative of the Baer product when $G / \theta_{G}(K)$ and $H / \theta_{H}(K)$ are isomorphic. It is a simple matter to verify that $\mathfrak{G}$ is a representative of the Baer product if and only if $\theta_{G}$ and $\theta_{H}$ are isomorphisms. In this case we obtain $\left(5=G, L_{1}(G) \otimes_{L_{1}(G)} L_{1}(G)=L_{1}(G)\right.$ a result derivable by direct examination of the data.

\title{
BIBLIOGRA PHY
}

1. R. Baer, Erweiterung von Gruppen und ihren Isomorphismen, Math. Z. 38 (1934), 375-416.

2. E. Cartan and S. Eilenberg, Homological algebra, Princeton Univ. Press, Princeton, N. J., 1956.

3. P. J. Cohen, Factorization in group algebras, Duke Math. J. 26 (1959), 199-206.

4. B. R. Gelbaum, Tensor products of Banach algebras, Canad. J. Math. 11 (1959), 297-310.

5. - Note on the tensor product of Banach algebras, Proc. Amer. Math. Soc. 12(1961), $750-757$.

6. - Tensor products and related questions, Trans. Amer. Math. Soc. 103 (1962), 525-548.

7. A. Grothendieck, Produits tensoriels topologiques et espaces nucléaires, Mem. Amer. Math. Soc. No. 16 (1955), 331 pp.

8. K. Hoffman, Banach spaces of analytic functions, Prentice-Hall, Englewood Cliffs, N. J., 1962.

9. G. P. Johnson, Spaces of functions with values in a Banach algebra, Trans. Amer. Math. Soc. 92 (1959), 411-429.

10. L. Loomis, Introduction to abstract harmonic analysis, Van Nostrand, New York, 1953.

\author{
Institute for Defense ANALYSES, \\ Princeton, New Jersey \\ UNIVERSITY OF MINNESOTA, \\ MinNEAPOLIS, MinNESOTA
}

